\title{
DONOR SITE MORBIDITY OF FIBULAR FLAP
}

\section{Ghazanfar Ali, Abdul Majid*, Danish Almas, Shahid Hameed*, Rizwan Aslam**, Zubair Ahmad Khan***}

Combined Military Hospital Multan/National University of Medical Sciences (NUMS) Pakistan, *Combined Military Hospital /National University of Medical Sciences (NUMS) Rawalpindi Pakistan, ${ }^{* *}$ Combined Military Hospital Kharian/National University of Medical Sciences (NUMS) Pakistan, ${ }^{* * * S a d i q ~ H o s p i t a l, ~}$ Sargodha Pakistan

\begin{abstract}
Objective: To present the early and late outcomes related to donor site morbidity of Free Fibular Flap.

Study Design: Prospective observational study.

Place and Duration of Study: Plastic surgery department, Combined Military Hospital Rawalpindi, from Jan 2009 to Jan 2020. Methodology: In total 361 patients were enrolled for the study through consecutive purposive sampling. All patients requiring free fibula flap for reconstruction of the defect were included in the study. However, the patients with Diabetes Mellitus, Smoking or peripheral vascular disease were excluded from the study. The patients were asked to visit on second week and then at three months for late outcomes. The results were entered SPSS-24 for analysis and interpretation of data.

Results: Out of total 361 patients enrolled for study $293(81.16 \%)$ were male and $68(18.83 \%)$ were female. The men age was 51.43 years SD 14.6 (range 4 year to 68 years). In early outcomes, graft loss $29(8.03 \%)$ and sensory deficit 19 (5.26\%), are the commonest adverse outcomes. Results of late follow up revealed chronic pain in 29 (8.03\%), ankle instability in 24 (6.64\%), gait abnormality in $29(8.76 \%)$ and claw toe in 33 (9.14\%) patients. The American Orthopedic Foot and Ankle Society (AOFAS) score was $88.45 \%$. Majority $(52.63 \%)$ patients were very satisfied.

Conclusion: The free Fibula is excellent choice for reconstruction of a wide range of osseous defects with minimum donor site morbidity and high satisfaction rate among the patients.
\end{abstract}

Keywords: Donor site, Free fibula, Morbidity.

This is an Open Access article distributed under the terms of the Creative Commons Attribution License (http://creativecommons.org/licenses/by/4.0), which permits unrestricted use, distribution, and reproduction in any medium, provided the original work is properly cited.

\section{INTRODUCTION}

Fibula is a long bone in the leg. Although it is non-weight bearing bone, yet it plays important role in stability of ankle mortis and knee joint. Moreover, there are many musclesin all the compartments of the leg which arise from the Fibula and their weakness or injury can impact the daily life of the patients ${ }^{1}$. Fibula gets its vascular supply from peroneal artery and anterior tibial artery. As the vascular pedicle has sufficient length and diameter, it can be anastomosed easily with recipient vessels, Because of excellent vascular supply; osteotomies can be performed as close as $1 \mathrm{~cm}$ without compromising blood supply. Being a tubular structure Fibula is an excellent source of osseous tissue for reconstruction of many osseous defects like Mandible². It has long bone with sufficient thickness and can be carved for giving mandibular shape to the fibula ${ }^{3,4}$. The Flexor Hallucis Longus Muscle located on its posterior border can be used for filling the dead space. The central part of the shaft of fibula can be harvested leaving distal $6 \mathrm{~cm}$ for stability of ankle mortis and proximal $4 \mathrm{~cm}$ for safety of common peroneal nerve and insertion of lateral collateral ligament of knee joint. However, all

Correspondence: Dr Ghazanfar Ali, Plastic Surgeon, Combined Military Hospital, Multan Pakistan

Received: 01 Sep 2020; revised received: 30 Dec 2020; accepted: 05 Jan 2021 of this comes at some cost, as there is no free lunch. First of all, the blood supply of skin paddle is unreliable in about $9 \%$ patients ${ }^{1}$. The Common Peroneal Nerve curves around the upper end hand hence vulnerable to injury leading to foot drop. Other problems include; wound infection, presence of long scar, gait abnormalities 5 .

Use of free Fibula flap dates back to 1975 when Taylor ${ }^{6}$ used it for reconstruction of large tibial defect. However, Hidalgo ${ }^{7}$ was the pioneer to report a large series using free fibula to reconstruct mandibular defects in 1989. After that, it became first choice for reconstruction of mandible and other osseous defects. The free fibula flap is commonly used for reconstruction of Mandible, other long bones and revascularization of avascular necrosis of head of femur-10. We used osteocutaneous as well as osseous flaps for bony reconstruction.

There is always a room for improvement, so we conducted a study to report our experience of using free fibula focusing on it donor site morbidity.

\section{METHODOLOGY}

This prospective observational study was conducted from Jan 2009 to Jan 2020 in plastic surgery department of Combined Military Hospital Rawalpindi. The study was conducted after approval from ethics com- 
mittee of the hospital. In total 361 patients were enrolled for the study through consecutive purposive sampling. All patients requiring free fibula flap for reconstruction of the defect were included in the study. However, the patients with Diabetes Mellitus, Smoking or peripheral vascular disease were excluded from the study due to higher chances of developing complications and posing higher risk for respiratory and cardiac complications under general anesthesia.

Pre-operatively, they were counseled about the nature of surgery, duration of hospital stay, follow ups and possible adverse outcomes.A detailed history was taken from the patients. History regarding the previous trauma, smoking, Diabetes Mellitus, Hypertension, Peripheral vascular disease, venous insufficiency and previous surgery was obtained and data was recorded on specially designed proforma. Detailed neurological, vascular and musculo-skeletal examination was carried out. Doppler ultrasound was used to mark the perforators. Detail of surgical procedure is given under separate heading (Surgical Procedure).

Post operatively, the patients were followed up on second week for early Outcomes and at three months for late outcomes. For early outcomes, the patients were examined for presence of Surgical Site Infection, Hematoma formation, Graft Loss, Tendon Desiccation and Sensory Deficit. For late outcomes, they were examined for presence of chronic pain, Ankle Instability, gait abnormality, Muscle weakness (Flexor Hallucis Longus) and Claw Toe. The patients were also assessed subjectively for their satisfaction about the donor site appearance. American Orthopedic Foot and Ankle Society Scoring were also done based on subjective assessment of patients. SPSS-24 was used for analysis and interpretation of data. The data were described as numbers and percentages. Variables used for this study were age, gender, indication for Free Fibula flap, early outcomes (Surgical Site infection, Hematoma formation, Graft Loss, Tendon Desiccation and sensory deficit),Late outcomes (Chronic Pain, ankle instability, gait abnormality, muscle weakness, Claw toe), AOFAS score and Patient satisfaction (very satisfied, satisfied, intermediate and unsatisfied).

All patients are operated under general anesthesia with tourniquet. The operation is carried out in supine position with knee flexed at 900 and hip joint rotated internally. After carrying out resection on the recipient area, the defect is assessed and reverse planning is done. The flap is marked on the donor site. The outline of Fibula, location of perforators, position of nutrient vessel and skin paddle (if required) are marked.

The skin incision is made on the lateral surface on the posterior inter-muscular septum and dissection is carried out anterior to this plane and peroneus long us and Brevis are lifted off the bone. The Lateral intermuscular septum is incised to enter the anterior compartment. Extensor Digitorum Longus and Extensor Hallucis Longus are lifted off the fibula and inter-osseous membrane is divided to enter the deep posterior compartment. The Peroneal vessel located between Tibialis Posterior and Flexor Hallucis Longus are carefully identified, dissected and saved. Osteotomies are performed for harvest the shaft of Fibula and distal $6 \mathrm{~cm}$ and proximal $4 \mathrm{~cm}$ of the fibula are preserved. The fibula is carved accordingto requirement by making osteotomies. The peroneal vessels are anastomosed with the recipient vessels (fig-1 \& 2).

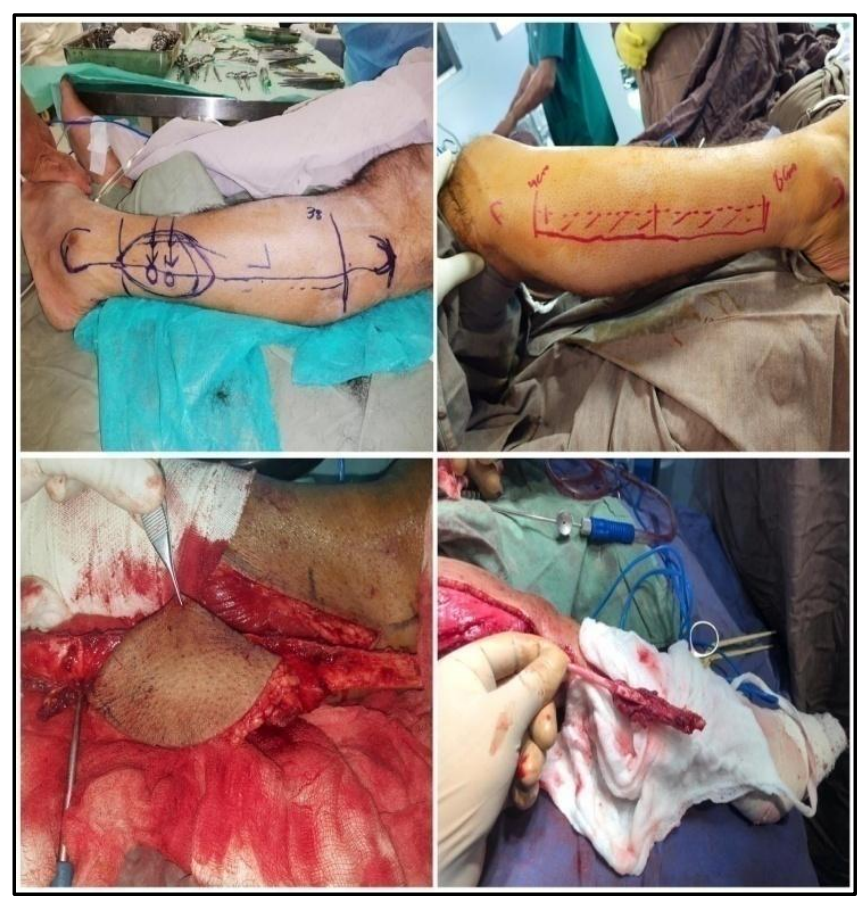

Figure-1: Marking for osteo-cutaneous flap (upper left), osseous flap (upper right), dissected osteo-cutaneous flap (lower left) and dissected osseous flap (lower right).

\section{RESULTS}

Out of total 361 patients enrolled for study 293 $(81.16 \%)$ were male and $68(18.83 \%)$ were female. The men age was 51.43 years, SD 14.6 (range 4-68 years). Reconstruction of mandible was commonest indication for Free Fibula Flap followed by Avascular Necrosis (AVN) Hip (table-I). In early outcome the Loss of Graft 
was commonest complication 29 (8.03\%) Patients, followed by sensory deficit in area of common peroneal nerve 19 (5.26\%) (table-II).

In late outcomes, the commonest complication was Flexor Hallucis Longus Muscle weakness 37 $(10.24 \%)$ followed by claw toe $33(9.14 \%)$ (table-III).

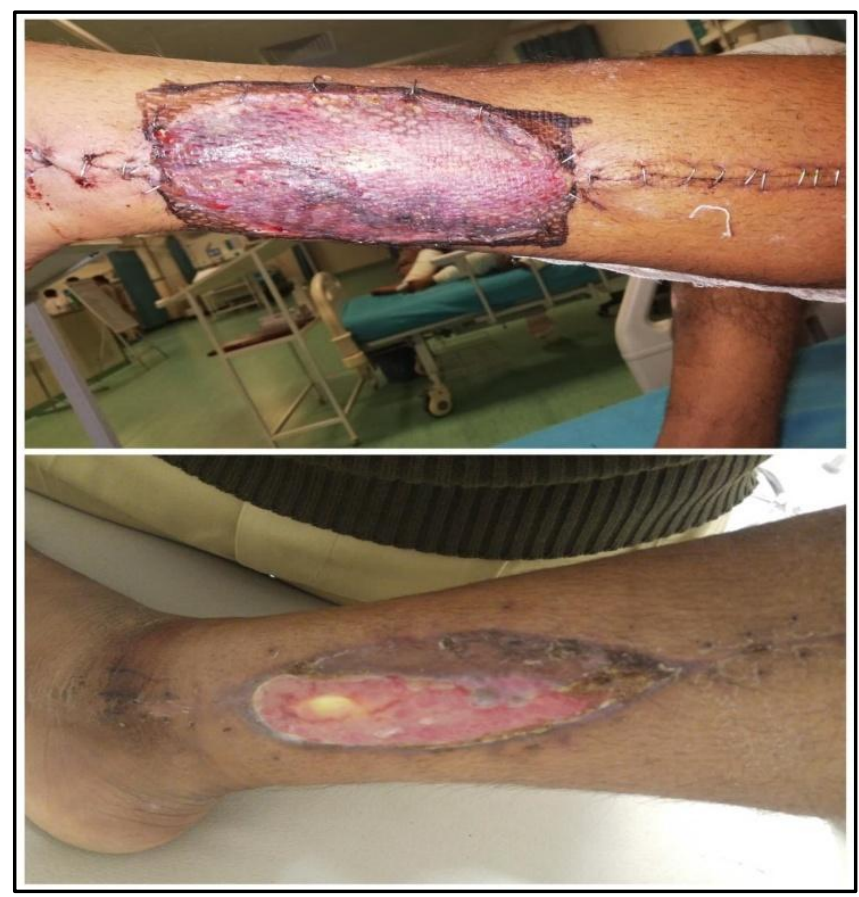

Figure-2: Normal skingraft (upper) and exposed tendon at donor site (lower).

The American Orthopedic Foot and Ankle Society (AOFAS) was $88.45 \%$. Results of Aesthetic outcome revealed that $190(52.63 \%)$ were very satisfied, 93 $(25.76 \%)$ were satisfied, $51(14.13 \%)$ were intermediate opinion and $27(7.48 \%)$ were unsatisfied.

\section{DISCUSSION}

Plastic surgeons are often consulted for the reconstruction of the defects which are otherwise difficult to reconstruct. As a problem solving Speciality, the plastic surgeons deal with the reconstruction of defects ranging from head to toe ${ }^{11}$. After analyzing the defects, the reconstructive ladder is followed and a suitable option is chosen for that particular problem in a particular patient. Use of free flap is an important tool in armamentarium of plastic and reconstructive surgeon and it immensely enhanced the options for reconstruction. The most important factors in selection of donor site are like for like and donor site morbidity. The ease of harvesting and position of patient are also important factors ${ }^{12,13}$.
Surgical site infection and hematoma formation are among the early complications. In our study the surgical site infection was noted in $18(4.98 \%)$ patients while hematoma formation was reported in $9(2.49 \%)$ patients. It is compatible with the contemporary studies. In a study conducted by Anthony et al ${ }^{14}$ in San

Table-I: Indications of free fibula flap.

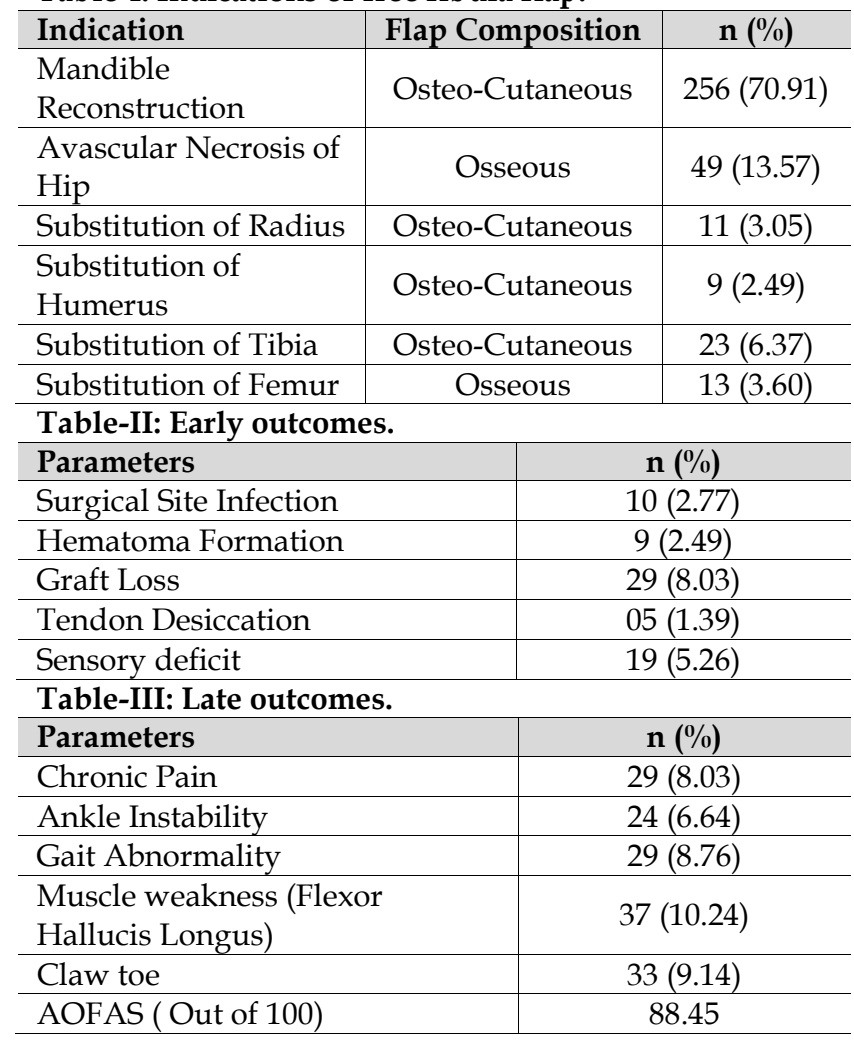

Francisco they reported cellulitis and wound dehiscence in $7 \%$ patients. In their study, the sample size was 29 and it is quite small as compared to our study. In another study conducted by Ling \& Peng ${ }^{15}$ conducted study on the same topic and reported wound infection in $9.9 \%$ patients and graft loss in $19 \%$ patients. Every effort should be made to minimize the infection rate as it is a clean surgery and surgical site infection may increase the cost as well as prolong the hospital stay of the patients.

In early outcome, sensory nerve injuries and graft loss add miseries to the life of patients. In our study, only $19(5.26 \%)$ patients had sensory deficit due to injury to common peroneal nerve and $29(8.03 \%)$ patients had Graft loss at donor site. Fortunately, 9 (2.49\%) patients of sensory nerve injuries recovered over a period of about six months. A study conducted by Shpitzer, Neligen and Boyd et al ${ }^{16}$ reported weakness of dorsiflexion in $4(10 \%)$ cases. This was because of 
injury to deep peroneal nerve or adhesions of the extensor muscles. They also reported weakness of planterflexion in $5(12 \%)$ patients. This weakness was due to deep peroneal nerve injury or injury to the flexor muscles. Zimmermann, Borner and Hasse et al ${ }^{17}$ conducted study on the same topic and they reported the incidence of objective sensory deficit as high as in $76 \%$ patients. In contrast to that study, Ling et al15 conducted study on the same topic and reported sensory deficit dueto common peroneal nerve injury in $21 \%$ patients and complain of cold intolerance in 10\% patients. These complications can be minimized with meticulous surgical technique, use of magnification loupes and to urniquet.

In late outcome parameters $37(10.24 \%)$ patients had weakness of the Flexor Hallucis Longus and 33 $(9.14 \%)$ had claw toe deformity. While $29(8.03 \%)$ had chronic pain and gait abnormality. There is wide range of parameters for gauging the outcomes regarding donor site morbidity. Bodde, De Visser, Duysens. et al ${ }^{18}$ use a table comprised of Point evaluation system to document the morbidity of donor site. The parameters used were; pain, walking ability, restriction in activities, gait alteration and cosmetic appearance. They used 0-4 points for these parameters. They used subjective and quantitative analysis methods and reported a feeling of ankle instability in 30\% patients and inability to run in $20 \%$ patients. It is quite a high number but their sample size was very small as compared to our study.

Gait Abnormality after harvesting middle part of fibula while leaving distal and proximal parts intact can be due to injury to leg muscles, nerves and local scarring. A study conducted by Ling and Peng ${ }^{15}$ on the donor site morbidity of free fibula flap reported a "considerable gait abnormality in 3.9\% patients." They also reported ankle instability in $5.8 \%$, claw toe in $6.1 \%$, and dorsiflexion of great toe in $3.6 \%$. A similar study conducted by Mojalla, Vayvada and Menderes et al ${ }^{19}$ reported ankle instability in $7 \%$ cases and $76 \%$ patients had restricted physical activity. There is wide variation among the results and they reflect diversity of patients and researchers.

Chronic pain at the donor site can be very problematic for patients. Ling and Peng ${ }^{15}$ reported presence of chronic pain in $6.5 \%$ patients in a study conducted on the donor site morbidity of fibula Flap. This is not a very significant percentage. There is variation in presence of chronic pain at donor site. Akashi, Hashikawa and Takasu et al20 in 2016 reported chronic pain in 20\% patients while Feuvrier, Sagawa, Beliard et al ${ }^{21}$ reported in $73 \%$ patients. This wide range of incidence shows that threshold of the patients may also vary.

Presence of scar poses a real aesthetic problem especially in women wearing short dress with exposed legs. A meticulous surgical technique, layered closure of wound and scar message can make the scar inconspicuous. Aesthetic assessment is mainly done through observation and difficult to standardize. Maciejewski and Szymcyk ${ }^{22}$ also used Self-rated donor site appearance for assessment of aesthetic outcomes for free fibula donor siteand reported $78 \%$ good/very good, $17 \%$ Moderate and 5\% poor Outcomes in their study. Other researchers used different assessment method and reported different results. Bodde et al ${ }^{18}$ used point evaluation system and reported 50\% excellent, $20 \%$ good, $10 \%$ moderate $10 \%$ intermediate and $10 \%$ bad outcome of aesthetic assessment. The patients were also assessed for AOFAS scores. It is different among different studies; Ling \& Peng ${ }^{15}$ reported AOFAS score 93.7\% while Mojallal et al ${ }^{19}$ reported AOFAS score $96.89 \%$. These scores are very similar to ours.

\section{ACKNOWLEDGEMENT}

The authors of this article acknowledge the valuable support provided by Abdul Rehman Ibrahim Bajwa for this scientific writing.

\section{CONCLUSION}

The free fibula is excellent choice for reconstruction of a wide range of osseous defects with minimum donor site morbidity and high satisfaction rate among the patients. However, cost of donor site morbidity should be considered.

\section{CONFLICT OF INTEREST}

This study has no conflict of interest to be declared by any author.

\section{REFERENCES}

1. Wei FC, Chen HC, Chuang CC, Noordhoff MS. Fibular osteoseptocutaneous flap: anatomic study and clinical application. Plast Reconstr Surg 1986; 78(2): 191-200.

2. Schardt C, Schmid A, Bodem J, Krisam J, Hoffmann J, Mertens C. Donor site morbidity and quality of life after microvascular head and neck reconstruction with free fibula and deep-circumflex iliac artery flaps. J Cranio-Maxillofacial Surg 2017; 45(2): 304-11.

3. Kearns M, Ermogenous P, Myers S, Ghanem AM. Osteocutaneous flaps for head and neck reconstruction: A focused evaluation of donor site morbidity and patient reported outcome measures in different reconstruction options. Arch Plastic Surg 2018; 45(6): 495.

4. Fang H, Liu F, Sun C, Pang P. Impact of wound closure on fibular donor-site morbidity: a meta-analysis. BMC Surg 2019; 19(1): 81. 
5. Xu ZF, Bai S, Zhang ZQ, Duan WY, Wang ZQ, Sun CF. A critical assessment of the fibula flap donor site. Head Neck 2017; 39(2): 279-87.

6. Taylor GI, Miller GD, Ham FJ. The free vascularized bone graft. A clinical extension of microvascular techniques. Plast Reconstr Surg 1975; 55(5): 533-44.

7. Hidalgo DA. Fibula free flap: a new method of mandible reconstruction. Plast Reconstr Surg 1989; 84(1): 71-79.

8. Taylor GI, Corlett RJ, Ashton MW. The evolution of free vascularized bone transfer: a 40-year experience. Plast Reconstr Surg 2016; 137(4): 1292-305.

9. Hadouiri N, Feuvrier D, Pauchot J, Decavel P, Sagawa Y. Donor site morbidity after vascularized fibula free flap: gait analysis during prolonged walk conditions. Intl J Oral Maxillofac Surg 2018; 47(3): 309-15.

10. Hadouiri N, Feuvrier D, Pauchot J, Decavel P, Sagawa Y. Donor site morbidity after vascularized fibula free flap: gait analysis during prolonged walk conditions. Intl J Oral Maxillofac Surg 2018; 47(3): 309-15.

11. Thorne $\mathrm{CH}$. Techniques and principles in plastic surgery. Grabb and Smith's Plastic Surgery. 6th ed. Philadelphia: LippincottRaven. 2007: 1-11.

12. Shroff SS, Nair SC, Shah A, Kumar B. Versatility of fibula free flap in reconstruction of facial defects: a center study. J Maxillofac Oral Surg 2017; 16(1): 101-07.

13. Hakim SG, Tehrany AS, Wendlandt R, Jacobsen HC, Trenkle T, Sieg $P$. The impact of harvest length and detachment of the interosseous membrane on donor-site morbidity following free fibula flap surgery - a biomechanical experimental study. J Craniofac Surg 2018; 46(11): 1939-42.
14. Anthony JP, Rawnsley JD, Benhaim P, Ritter EF, Sadowsky SH Singer MI. Donor leg morbidity and function after fibula free flap mandible reconstruction. Plast Reconstr Surg 1995; 96(1): 146-52.

15. Ling XF, Peng $X$. What is the price to pay for a free fibula flap? A systematic review of donor-site morbidity following free fibula flap surgery. Plast Reconstr Surg 2012; 129(3): 657-74.

16. Shpitzer T, Neligan P, Boyd B, Gullane P, Gur E, Freeman J. Leg morbidity and function following fibular free flap harvest. Ann Plast Surg 1997; 38(5): 460-64.

17. Zimmermann C, Börner BI, Hasse A, Sieg P. Donor site morbidity after microvascular fibula transfer. Clin Oral Investig 2001; 5(4): 214-19.

18. Bodde EW, De Visser E, Duysens JE, Hartman EH. Donor-site morbidity after free vascularized autogenous fibular transfer: subjective and quantitative analyses. Plast Reconstr Surg 2003; 111(7): 2237-42.

19. Mojallal A, Besse JL, Breton P. Donor site morbidity after free fibula flap. Report of 42 consecutive cases. Ann Chir Plast Esthet 2004; 49(1): 3-10.

20. Akashi M, Hashikawa K, Takasu H, Watanabe K, Kusumoto J, Sakakibara A, et al. Comparison between primary closure and skin grafts of the free fibula osteocutaneous flap donor site. J Oral Maxillofac Surg 2016; 20(3): 233-37.

21. Feuvrier D, Sagawa Y, Béliard S, Pauchot J, Decavel P. Long-term donor-site morbidity after vascularized free fibula flap harvesting: clinical and gait analysis. J Plast Reconstr Aesthet Surg 2016; 69(2): 262-69.

22. Maciejewski A, Szymczyk C. Fibula free flap for mandible reconstruction: analysis of 30 consecutive cases and quality of life evaluation. J Reconstr Microsurg 2007; 23(01): 001-10. 